occupational OP exposure and deficits on behavioural performance tests. We studied pesticide application teams in Egypt who are primarily exposed to one OP, chlorpyrifos $(\mathrm{CPF})$, differing in exposure experience based on their job category.

Methods Trailmaking A and B were administered to 54 engineers (who typically watch the applications from beside the field), 59 technicians (who typically guide the applicators in the field), 31 applicators (using knapsack sprayers), and 150 controls (who did not work in the fields) at 2 different times during the OP application season and also immediately and 1.5 months after applications had ended.

Results A consistent dose-response relationship was seen in performance speed: Controls had the best performance through most of the application season on Trailmaking A $(\mathrm{p} \leq 0.04)$ and B $(\mathrm{p}<0.001)$. Applicators had slower performance than engineers $(p=0.015)$ and technicians $(p=0.032)$. On the more complex Trailmaking B test, applicators and technicians had comparable performance that was significantly slower $(p=0.003$ and $p=0.012$ respectively) than performance of the engineers. Test performance at 1.5 months after applications ended and in the following year revealed that differences between the groups were persistent, and some differences were significant. 3,5,6-Trichloro-2-pyridinol (TCPy) levels in urine confirmed the pattern of higher to lower exposures across the job categories of the pesticide application teams, and these were all greater than exposures in controls. Increasing TCPy concentrations were significantly correlated with slower Trailmaking B performance at 1.5 months after the exposures had ceased, but not during or immediately after exposures.

Conclusion This study identifies a dose-response based on job category and establishes that the OP chlorpyrifos is neurotoxic.

\section{9d PRECLINICAL MODEL OF CHLORPYRIFOS EXPOSURES AND EFFECTS DOCUMENTED IN EGYPTIAN PESTICIDE APPLICATORS}

PJ Lein. Molecular Biosciences, University of California, Davis, CA, USA

\subsection{6/oemed-2018-ICOHabstracts.822}

Introduction Organophosphorus pesticide (OP)-induced neurotoxicity remains a significant occupational health concern, management of which is complicated by the lack of biomarkers that reliably identify at-risk individuals. To address this issue, we used a preclinical model of occupational OP exposure to evaluate the relationship between OP-induced cognitive deficits and expression of conventional and novel biomarkers of exposure and effect.

Methods Adult male Long Evans rats were exposed to CPF (3 or $10 \mathrm{mg} / \mathrm{kg} / \mathrm{d}$, s.c.) or an equal volume of vehicle for up to 21 days. Previous PBPK modelling studies confirmed that this exposure paradigm resulted in urinary TCPy levels and blood cholinesterase activity within the range of those observed in exposed Egyptian pesticide applicators. Learning and memory were assessed using appetitive Pavlovian discrimination between two tones and by Pavlovian fear conditioning. Tissues were collected for quantification of peripheral and central biomarkers of CPF exposure, inflammation and oxidative stress.

Results Subchronic CPF exposure for $21 \mathrm{~d}$ interfered with the maintenance and reversal of appetitive behaviour, but these effects were not reliable. In contrast, CPF caused robust and reproducible dose-dependent deficits in Pavlovian fear conditioning at 21 but not 4, 10 or 15 days of exposure. CPF also increased urinary TCPy levels, caused a delayed decrease in blood and brain cholinesterase activity, increased urinary and brain F2-isoprostanes and upregulated expression of multiple oxidative stress biomarkers in brain and in the periphery. Of these biomarkers, only oxidative stress biomarkers correlated with cognitive deficits. Moreover, CPF-induced cognitive deficits were prevented by co-administration of the antioxidant Trolox (1 mg/kg, i.p.).

Conclusion These findings demonstrate that subchronic exposures to CPF at levels that do not cause systemic cholinergic toxicity impair learning and memory via effects on the amygdala and hippocampus. Biomarker analyses suggest that oxidative stress, but not cholinesterase inhibition, contribute to CPF-induced cognitive deficits.

Funding: R01 ES016308

\section{9e EFFECTIVENESS OF EXPOSURE PREVENTION CLOTHING IN THE EGYPTIAN APPLICATORS THAT COULD BE IMPLEMENTED WITH MINIMAL COST}

FM Farahat. Menoufia University, Egypt

\subsection{6/oemed-2018-ICOHabstracts.823}

Introduction Chlopyrifos organophosphorous pesticides (OPs) are regularly applied for protection of the cotton crop in Egypt. OP absorption subsequent to dermal exposure has been estimated to be $94 \%-96 \%$ of the total dose. Legs and lower body parts are the most affected during cotton pesticide applications. Pesticides residues on the cotton plants also contaminate clothing and uncovered arms or legs of the applicators as they pass. Protective equipment is not readily available in Egypt. This pilot study was conducted to determine if wearing plastic coverings over pants or changing work practices could reduce pesticide exposure among Egyptian pesticide workers.

Methods A controlled intervention study included:

- protective clothing (plastic pants modelled by the workers and staff who participated in 4 educational focus groups);

- spray away (instructions were provided to spray away form the workers' path);

- control (followed routine work practices).

Exposure was assessed measuring pre- and post-application urinary TCPy levels (3,5,6-trichloro-2-pyridinol), the primary chlorpyrifos metabolite used as a biomarker of absorbed dose. Work activities were recorded throughout the three-day study period.

Results Twenty-four adult workers ( $\mathrm{n}=8$ per group) participated in the study. Time spent applying (range 15-36 min) and mixing (range 10-12 min) pesticides varied between groups and job categories. Other than the pants group, none of the workers had protective clothing. Spraying away did not produce consistent results. Average TCPy levels of the protective clothing group were lower compared to the other two groups, though the differences were not statistically significant $(\mathrm{p}>0.05)$.

Conclusion Work habits, time spent applying or mixing pesticides and environmental conditions (e.g., wind direction) are important exposure determinants of urinary TCPy levels. However, use of protective clothing covering legs and lower 